\title{
Was denken linguistische Laien über Sprache?
}

\section{Ergebnisse einer aktuellen Repräsentativerhebung zu Spracheinstellungen in Deutschland}

Zusammenfassung: Öffentliche Sprachdiskurse, wie sie beispielsweise in den Medien stattfinden, werden typischerweise aus einer sprachkritischen Haltung heraus geführt. Inwieweit diese veröffentlichte Meinung tatsächlich die Mehrheitsmeinung der Sprecherinnen und Sprecher widerspiegelt, ist durchaus eine offene Frage. In diesem Beitrag berichten wir aus einer rezenten Erhebung über Spracheinstellungen in Deutschland. Wir zeigen, dass die Art der Frageformulierung einen starken Einfluss auf die Ergebnisse hat, und berichten, welche sprachlichen Veränderungen die Befragten in jüngerer Zeit angeben, wahrgenommen zu haben.

Abstract: Discourse about language in the public arena is typically conducted in an attitude of language criticism. It remains questionable, in how far this publicly issued opinion actually reflects the opinion of the majority of speakers. In this contribution we report the results of a recent survey on language attitudes in Germany. We show that the wording of the question has a strong impact on the results. Also, we evaluate the linguistic changes that respondents report to have noticed in recent time.

Schlüsselwörter: Anglizismen, Mehrsprachigkeit, Spracheinstellungen, Sprachwandel, anglicisms, multilingualism, language attitudes, language change

Adler, Astrid: Leibniz-Institut für Deutsche Sprache, Augustaanlage 32, 68165 Mannheim, adler@ids-mannheim.de

Plewnia, Albrecht: Leibniz-Institut für Deutsche Sprache, Augustaanlage 32, 68165 Mannheim, plewnia@ids-mannheim.de 


\section{Einleitung}

Öffentliche Diskurse über Sprache, wie sie beispielsweise in Leserbriefspalten (nicht selten induziert durch einschlägige Feuilleton-Artikel), in den Kommentarbereichen von Online-Nachrichtenportalen oder in verschiedensten InternetForen stattfinden, weisen, bei aller Heterogenität im Detail, einige typische Merkmale auf. So lässt sich zum Beispiel zuverlässig beobachten, dass Meinungsfreudigkeit und fehlende Sachkunde einander keineswegs ausschließen (man ist fast geneigt zu sagen: im Gegenteil). Vor allem aber ist die Art, wie linguistische Laien über Sprache reden, überwiegend von großer Skepsis geprägt; es handelt sich typischerweise um einen Negativ-Diskurs (vgl. dazu Plewnia \& Witt 2014). Dabei dominiert ein Sprachverfalls-Topos, der so alt ist wie das Nachdenken über Sprache selbst (und der sich wiederum in einen allgemeineren Negativ-Diskurs um einen generellen Bildungs- und Sitten-Verfall einordnet); diesem Sprachverfalls-Diskurs untergeordnet sind einzelne Teildiskurse, beispielsweise zu Fragen von Sprachrichtigkeit (vorwiegend in Bezug auf grammatische Probleme), zu fremdsprachigen Einflüssen aufs Deutsche (hier geht es in erster Linie - aber nicht nur - um Anglizismen), zu bestimmten Varietäten (wie zur Jugendsprache) oder Fachsprachen (wie zur Sprache der Werbung), wobei diese Themenfelder natürlich nicht trennscharf sind, sondern sich in verschiedener Weise überlappen. Flankiert wird dieser allgemeine Sprachverfalls-Diskurs durch einen Diskurs über eine politische Dimension des Sprachgebrauchs, die üblicherweise mit dem Label political correctness belegt wird; auch dieser Diskurs ist stark durch einen Hang zu Polarisierungen und weniger durch Sachlichkeit geprägt.

Die hier angesprochene Negativ-Tendenz sei am Beispiel des AnglizismenDiskurses kurz illustriert. Eine erste Annäherung bietet eine einfache Kookkurrenzanalyse für das Lemma Anglizismen im Deutschen Referenzkorpus (DEREKO) des IDS. ${ }^{1}$ Ermittelt werden auf diese Weise diejenigen Wörter (Wortformen), die im Korpus mit überstatistischer Häufigkeit als Partnerwörter des Basislemmas auftreten. Visualisieren lässt sich das Ergebnis der Kookkurrenzanalyse zum Beispiel über eine Wortwolke (vgl. Abb. 1); relevantere Partnerwörter erscheinen in der Darstellung größer.

1 Vgl. https://www1.ids-mannheim.de/kl/projekte/korpora/ (letzter Zugriff 27.04.2020). 


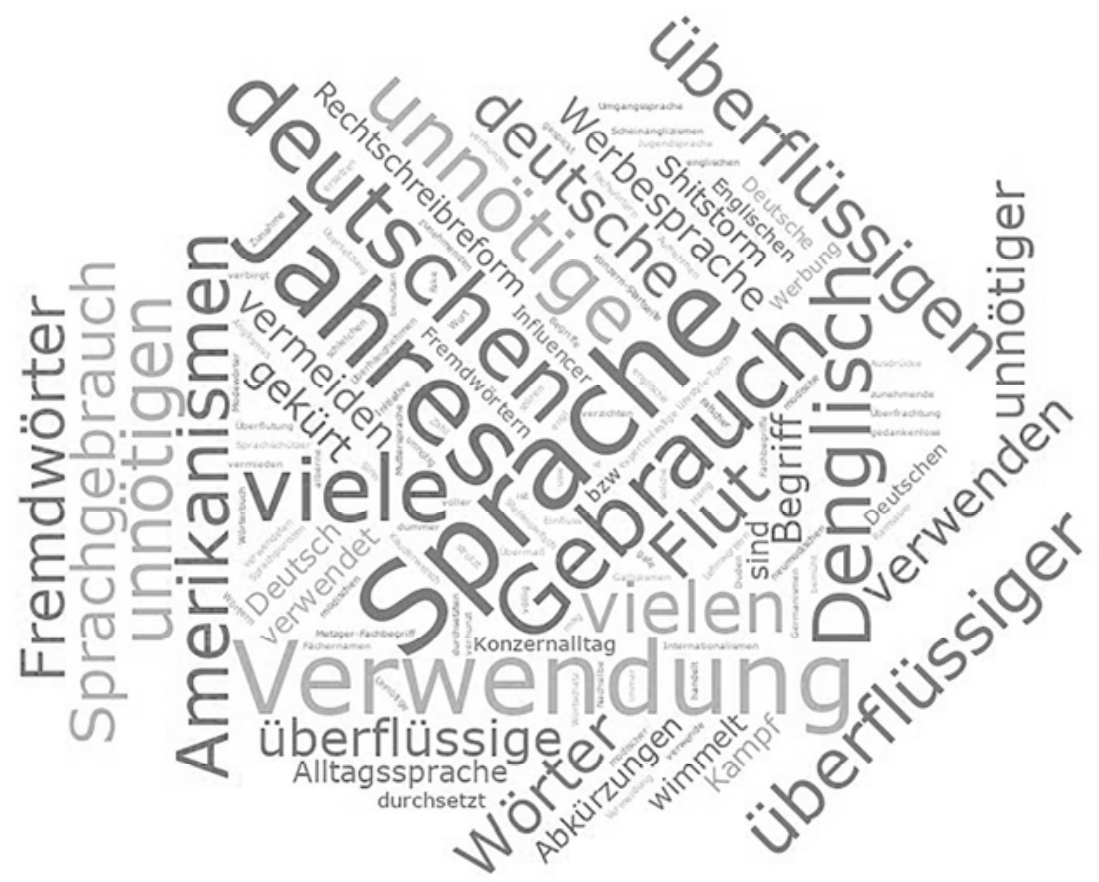

Abb. 1: Wortwolke Anglizismen.

Es wird unmittelbar augenfällig, wie sehr dieser negative Zungenschlag die Art und Weise, in der über Anglizismen gesprochen wird, dominiert - und zwar selbst in den ja überwiegend redigierten Texten professioneller Schreiber im DEREKO. Die vorherrschenden Attribute sind „überflüssig“ und „unnötig“ (jeweils in verschiedenen Flexionsformen), neben weiteren Negativzuschreibungen (,alberne“, „modische“, „dummer“, „hässlicher“, „unsinnigen“); viele Wörter benennen allgemein als zu groß bewertete Quantitäten (,wimmelt“, „strotzt“, „Überhandnehmen“, „übermäßigen“, „gespickten“), viele Wörter entstammen einer Metaphorik, die auf Naturkatastrophen (,Flut“, „Überflutung“) oder Seuchen (,durchsetzt“) verweist, viele Wörter zielen auf Distanzierung (,vermeiden“, „Vermeidung“, „verzichtet“, „Kampf“). 


\section{Zustand und Entwicklung des Deutschen}

Was hier schlaglichtartig und stark verkürzt für den öffentlichen AnglizismenDiskurs angedeutet wurde, ${ }^{2}$ lässt sich mutatis mutandis auch für die anderen angesprochenen Teildiskurse zeigen: dass öffentliche Sprachdiskurse fundamental von einer sprachskeptischen und sprachkritischen Haltung geprägt sind. Es ist allerdings eine interessante und durchaus offene Frage, inwieweit dieser (hier in sehr vergröberter Form vorgetragene) Befund für die gesamte Sprachgemeinschaft generalisierbar ist, das heißt, in welchem Maße dieser Charakter öffentlicher Sprachdiskurse auch die Mehrheitsmeinung der Sprecherinnen und Sprecher spiegelt. Es ist ja durchaus denkbar, dass die Debattenbeiträge nicht repräsentativ sind für das, was es in der Bevölkerung an Meinungen zu Sprache und Sprachgebrauch gibt, und dass sich an den öffentlichen Debatten nur oder zumindest vorwiegend die Unzufriedenen beteiligen, die dann mit ihrer Unzufriedenheit den Diskurs dominieren.

Ein möglicher Weg, das herauszufinden, führt über die quantitative Spracheinstellungsforschung. Der Ansatz besteht darin, sehr viele Menschen zu verschiedenen sprachlichen Fragen zu befragen und so mittels sehr großer, im Idealfall repräsentativer, Stichproben ein umfassendes Meinungsbild zum erhobenen Thema zu gewinnen; liegen bestimmte soziodemographische Angaben zur Stichprobe vor, lassen sich überdies mit statistischen Methoden die Profile bestimmter Typen von Befragten ermitteln. Eine entscheidende Rolle spielt bei diesem Vorgehen (wie in der quantitativen Sozialforschung überhaupt und bei Meinungsumfragen generell) die Art der Frageformulierung. Die Herausforderung, Fragen so zu formulieren, dass sie das erheben, was sie erheben sollen, ist keineswegs trivial; vor allem geht es darum, ein Priming der Gewährspersonen zu vermeiden (vgl. etwa Lenzner \& Menold 2015). ${ }^{3}$

Es gibt einige einschlägige Befragungen aus den letzten Jahren; wegen der Unterschiede in den Frageformulierungen sind sie jedoch nur eingeschränkt vergleichbar. Die Gesellschaft für deutsche Sprache (GfdS) gibt in unregelmäßigen Abständen Meinungsumfragen unterschiedlicher Tiefe $\mathrm{zu}$ verschiedenen sprachbezogenen Themen in Auftrag. Eine größere Umfrage zu Meinungen zur deutschen Sprache wurde für die GfdS im Jahr 2007 durch das Institut für De-

2 Zur sachlichen Fundierung dieses Diskurses vgl. z. B. Eisenberg (2013); Spitzmüller (2020). 3 Eine weitere, den Erhebungen nachgeordnete Herausforderung besteht darin, bei der Auswertung der Daten sicherzustellen, dass nur das als Ergebnis berichtet wird, was die Daten entsprechend den zugrunde liegenden Frageformulierungen auch hergeben. Insbesondere in journalistischen Texten gelingt das nicht immer. 
moskopie Allensbach durchgeführt (vgl. Hoberg, Eichhoff-Cyrus \& Schulz 2008). ${ }^{4}$ Eine der dort gestellten Fragen bezog sich explizit auf den Sprachverfalls-Diskurs (Abb. 2).

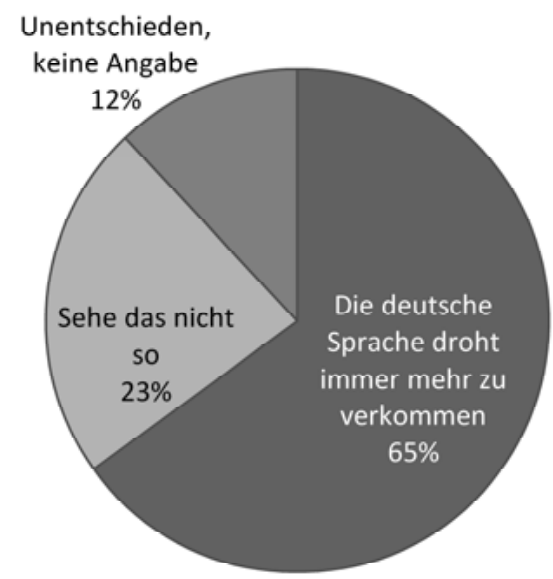

Abb. 2: „Die meisten befürchten, dass die deutsche Sprache immer mehr verkommt“5 (IfDAllensbach 2007; $N=1.820$; Frage: „Wenn jemand sagt: ,Die meisten Menschen bei uns in Deutschland legen nur noch wenig Wert auf eine gute Ausdrucksweise. Die deutsche Sprache droht immer mehr zu verkommen. "Sehen Sie das auch so, oder sehen Sie das nicht so?").

Auf den ersten Blick scheint sich hier die beschriebene allgemeine NegativTendenz öffentlicher Sprachdiskurse abzubilden: 65\%, fast zwei Drittel der Befragten, stimmt der angebotenen Aussage zu, nur $23 \%$, ein knappes Viertel, widerspricht. Allerdings wird man nicht umhinkommen zu konstatieren, dass die Frageformulierung nicht frei ist von einer gewissen Suggestivität. Die Formulierung ist nicht neutral, sie enthält implizite Wertungen (,Wert legen auf gute Ausdrucksweise“) und legt die präferierte Antwort nahe (Zustimmung ist immer leichter als Widerspruch). Das setzt sich im Folgenden fort: diejenigen, die mit „sehe ich auch so“ geantwortet haben, werden anschließend gefragt: „Woran liegt es Ihrer Meinung nach, dass die deutsche Sprache immer mehr verkommt?“ Das ist nun aber eine Präsupposition: Aus der Zustimmung zu

4 Es handelte sich um eine mündlich-persönlich durchgeführte Repräsentativbefragung der deutschen Wohnbevölkerung $(N=1.820)$.

5 Mit dieser Formulierung ist das entsprechende Diagramm in Hoberg, Eichhoff-Cyrus \& Schulz (2008: 10) übertitelt; dies entspricht nicht genau dem Wortlaut der Fragestellung. 
einem Zitat, die deutsche Sprache drohe immer mehr zu verkommen, wird hier eine Tatsachenbehauptung: die deutsche Sprache verkommt. Folgerichtig werden auch die Antworten (es werden sieben Möglichkeiten vorgegeben, vom Einfluss anderer Sprachen über digitale Kommunikation, Fernsehen und schlechten Schulunterricht) eingeleitet mit der Formulierung „Ich glaube, dass die deutsche Sprache verkommt, weil...“ (Hoberg, Eichhoff-Cyrus \& Schulz 2008: 11). Das ist methodisch nicht ganz sauber.

Eine ähnliche Erhebung mit einer ähnlichen Formulierung wurde im Jahr 2019 vom Erfurter Institut INSA-CONSULERE für die Theo-Münch-Stiftung für die Deutsche Sprache durchgeführt; ${ }^{6}$ sie erbrachte auch ähnliche Ergebnisse (Abb. 3).

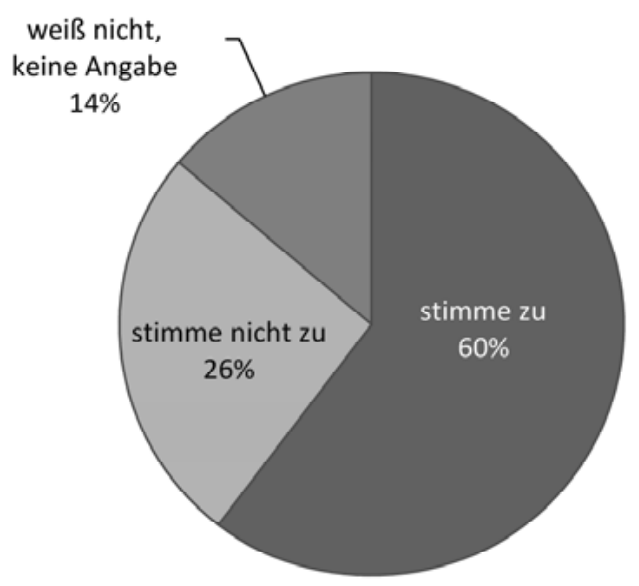

Abb. 3: „Die deutsche Sprache verkommt zunehmend.“ (INSA 2019; $N=2.079){ }^{7}$

Hier wird anscheinend in der Frageformulierung auf das Hedging verzichtet; ${ }^{8}$ die Werte für Zustimmung zur vorgelegten Behauptung (60\%) und Widerspruch ( $26 \%$ ) entsprechen in etwa denjenigen der zwölf Jahre älteren Allensbach-Umfrage.

6 Die Erhebung war Teil des sog. „INSA-Meinungstrends“ für den „BILD-Wahlcheck“ (N=2.079). Zur Umfrage vgl. Paulwitz (2019); Informationen finden sich auch auf der Website des „Vereins für Sprachpflege“ unter https://deutsche-sprachwelt.de/kategorie/insabefragung-zur-deutschen-sprache-2019-20/ (letzter Zugriff 15.6.2020).

7 Vgl. Paulwitz (2019).

8 Der vollständige Wortlaut des Fragebogens ist nicht verfügbar. 
Seit einigen Jahren befasst sich auch dass IDS verstärkt mit Spracheinstellungen. ${ }^{9}$ In der Deutschland-Erhebung 2008, einer gemeinsam mit der Universität Mannheim im Jahr 2008 durchgeführten, sehr umfassenden Erhebung zu Spracheinstellungen in Deutschland ${ }^{10}$ wurde ebenfalls der SprachverfallsDiskurs adressiert, allerdings wurde hier eine neutralere Formulierung gewählt, indem nach der „derzeitigen Entwicklung der deutschen Sprache“ gefragt wurde (Abb. 4).

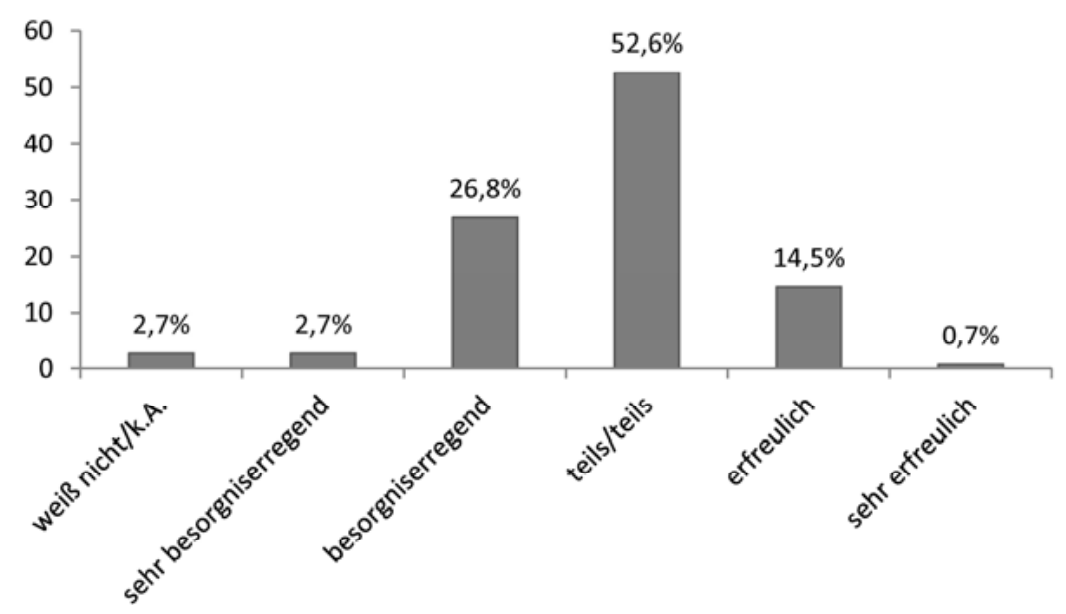

Abb. 4: Bewertung der Entwicklung der deutschen Sprache (DE2008; $N=2004$; Frage: „Einmal alles zusammen genommen: Finden Sie die derzeitige Entwicklung der deutschen Sprache ...").

Hier relativiert sich das sehr negative Bild, das sich aus den Fragen, die mit dem Terminus „verkommen“ operieren, ergeben hat. Nur unter $30 \%$ der Befragten geben an, sie fänden die derzeitige Entwicklung „,besorgniserregend“ oder „sehr besorgniserregend“, mehr als die Hälfte antwortet mit „teils/teils“ - worin zwar eine gewisse Skepsis zum Ausdruck kommt, die aber nicht dominiert. ${ }^{11}$ Allerdings ist anzumerken, dass hier, wiewohl die Frage selbst neutral formuliert

9 Zur aktuellen Spracheinstellungsforschung am IDS vgl. Adler \& Plewnia (2018).

10 Es handelte sich um eine telefonische Repräsentativerhebung unter der deutschen Wohnbevölkerung $(N=2.004)$, deren praktische Durchführung in den Händen der Forschungsgruppe Wahlen lag. Für einen Überblick über die Erhebung vgl. Eichinger et al. (2009), für detaillierte Ergebnisse Gärtig, Plewnia \& Rothe 2010; zu Einzelaspekten vgl. Plewnia \& Rothe (2009), zu weiteren Projektergebnissen vgl. Eichinger et al. (2012).

11 Für weitere Auswertungen dieser Frage vgl. Gärtig, Plewnia \& Rothe 2010: 212-214. 
war, die vorgegebenen Antwortoptionen (eine Fünfer-Skala von „sehr erfreulich“ bis „sehr besorgniserregend“) möglicherweise nicht optimal gewählt waren; der Terminus „besorgniserregend“ könnte durchaus auch ein gewisses Priming transportiert haben. ${ }^{12}$

Dass die Frageformulierung eine entscheidende Rolle spielt, konnten wir auch in unserer Deutschland-Erhebung 2017 zeigen. Die Deutschland-Erhebung 2017 wurde im Winter 2017/2018 vom IDS in Kooperation mit dem Deutschen Institut für Wirtschaftsforschung (DIW) im Rahmen der Innovationsstichprobe des Sozio-ökonomischen Panels (SOEP-IS) des DIW durchgeführt. ${ }^{13}$ Die Erhebung besteht aus zwei Teilen: einer direkten Befragung durch Interviewer ( $N=4.339)$ sowie einer anschließenden optionalen Zusatzbefragung mittels eines Online-Fragebogens ( $N=1.439)$. Die Gesamtstichprobe der Deutschland-Erhebung 2017 ist repräsentativ, für die Teilstichprobe der Zusatzbefragung gilt das mit gewissen Einschränkungen. Die Frage nach der Entwicklung der deutschen Sprache wurde auch im Online-Fragebogen der Deutschland-Erhebung 2017 gestellt. Die Frageformulierung orientierte sich an derjenigen von 2008, allerdings waren die Optionen auf der vorgegebenen Antwortskala von „sehr positiv“ bis „sehr negativ“ neutraler gefasst. Vor allem aber gab es hier als methodischen Kniff einen Split in der Stichprobe: die eine Hälfte der Probanden wurde, wie 2008, nach der „Entwicklung der deutschen Sprache“ gefragt, die andere Hälfte nach dem „aktuellen Zustand der deutschen Sprache“. Das Ergebnis zeigt auf eindrucksvolle Weise den Einfluss der Frageformulierung (Abb. 5).

$12 \mathrm{Zu}$ ähnlichen Ergebnissen kam auch eine bereits 1999 vom IDS durchgeführte Repräsentativbefragung ( $N=2.025$, mit einer Überquotierung Ostdeutschlands; vgl. Stickel \& Volz 1999). Die Frageformulierung lautete damals „Finden Sie die derzeitige Entwicklung der deutschen Sprache eher erfreulich oder eher besorgniserregend?“; 26,5\% der Befragten antworteten mit „eher besorgniserregend“, 26,2 \% „weder, noch“, 30,8 \% mit „teils, teils“ und 4,7 \% mit „eher erfreulich“ (Stickel \& Volz 1999: 21; für eine detaillierte Auswertung dieser Daten vgl. Pfalzgraf \& Leuschner 2006). - Auch in der INSA-Erhebung von 2019 wurde nach der Entwicklung gefragt: Die Frage „Wie beurteilen Sie allgemein die derzeitige Entwicklung der deutschen Sprache?“ beantworteten $27 \%$ der Befragten mit „sehr positiv“ oder „eher positiv“, $32 \%$ mit „teils, teils“ und 37 \% mit „eher negativ“ oder „sehr negativ“ (Paulwitz 2019: 3).

13 Für Einzelheiten zur Erhebung vgl. Adler \& Hansen 2020. 


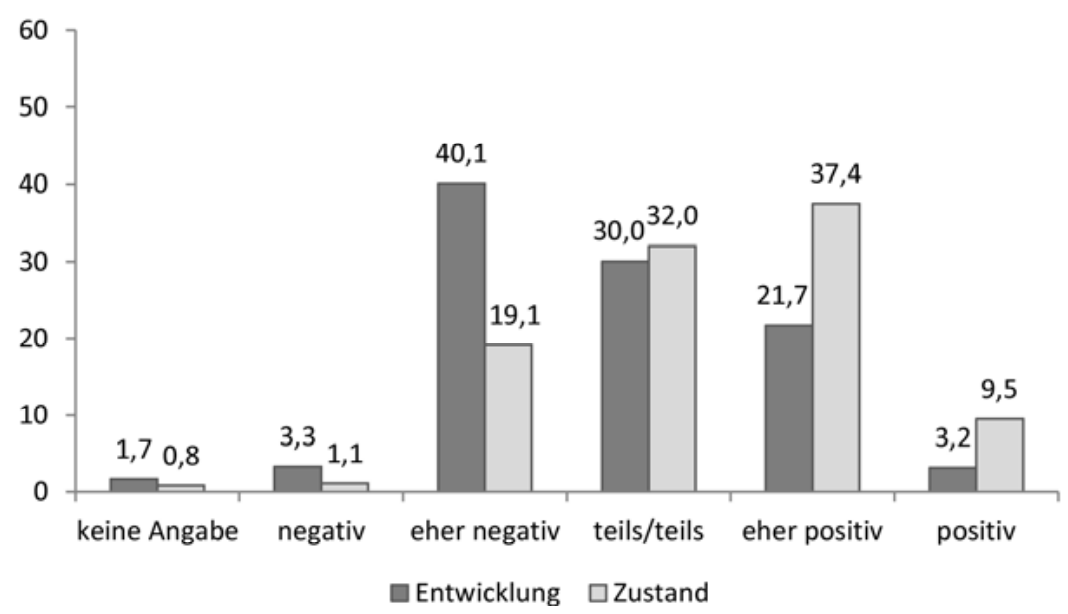

Abb. 5: Bewertung der Entwicklung bzw. des Zustands der deutschen Sprache (in Prozent; DE2017; $n_{\text {Entwicklung }}=723, n_{\text {Zustand }}=716$; Fragen: „Einmal alles zusammen genommen: Wie bewerten Sie die derzeitige Entwicklung der deutschen Sprache?“ bzw. „Einmal alles zusammen genommen: Wie bewerten Sie den aktuellen Zustand der deutschen Sprache?").

Tatsächlich wird der Zustand der deutschen Sprache (die rechten, hellen Balken) wesentlich weniger negativ bewertet als ihre Entwicklung (die linken, dunklen Balken).$^{14}$ Bei der Frage nach der Entwicklung dominieren mit rund $43 \%$ klar die negativen Bewertungen; nur ein Viertel der Befragten bewertet die Entwicklung als „eher positiv“ oder „positiv“. ${ }^{15}$ Ein genau komplementäres Bild ergibt sich für die Frage nach dem Zustand: hier gibt nur jeder fünfte Befragte ein negatives Urteil ab, fast die Hälfte (48 \%) bewertet den Zustand als „eher positiv“ oder „positiv“. (Die indifferente Mittelkategorie „teils/teils“ wird bei beiden Frageformulierungen von knapp jedem dritten Befragten - 30 bzw. 32 \% - ausgewählt.) Dieser Befund könnte auf eine gewisse Schlagseite des öffentlichen Diskurses hindeuten. Natürlich bedeutet eine unterschiedliche Bewertung

14 Als Grundlage für die in diesem Beitrag getroffenen Aussagen, die den Stellenwert von Variablen oder Unterschiede zwischen Variablen betreffen, wurden statistische Verfahren durchgeführt. Um den Text jedoch lesbar und allgemein verständlich zu halten, werden hier keine Details dieser Verfahren und statistische Kennwerte berichtet. Alle daran interessierten Leserinnen und Leser werden um eine persönliche Nachricht gebeten.

15 Dieses Ergebnis passt auch zur INSA-Umfrage; dort gab es auf die Frage „Wie beurteilen Sie allgemein die derzeitige Entwicklung der deutschen Sprache?“ folgende Antworten: $9 \%$ „sehr positiv“, 18 \% „eher positiv“, 32 \% „teils/teils“, 27 \% „eher negativ“, 10 \% „sehr negativ“, $5 \%$ „weiß nicht/keine Angabe“ (Paulwitz 2019: 3). 
von Zustand und Entwicklung zu einem gegebenen Zeitpunkt zunächst einmal keinen logischen Widerspruch. Der tritt freilich dann auf, wenn sich diese Diskrepanz der Bewertungen über die Zeit als stabil erweisen sollte: Wäre die Entwicklung fortwährend negativ, müsste sich ja auch der Zustand irgendwann wahrnehmbar verschlechtern. ${ }^{16}$

Eine interessante Frage ist nun, ob sich für diese beiden unterschiedlichen Frageformulierungen bei den Befragten auf der Ebene der soziodemographischen Merkmale unterschiedliche Muster zeigen. Wir demonstrieren das für die Merkmale ,Geschlecht', ,Alter' und ,formaler Bildungsabschluss' (Abb. 6a und 6b). Die Abbildungen zeigen für die jeweilige Teilstichprobe die Mittelwerte. Der Wert 0 entspricht dabei der Antwort „teils/teils“, der Wert 1 entspricht der Antwort „eher positiv“, der Wert -1 der Antwort „eher negativ“. Die Teilstichproben sind ihrerseits in zwei Gruppen geteilt: eine Gruppe mit dem jeweiligen Merkmal in niedriger Ausprägung (der jeweils linke, dunkle Balken) und eine Gruppe mit dem jeweiligen Merkmal in hoher Ausprägung (der jeweils rechte, helle Balken). ${ }^{17}$

16 Ein nicht ganz paralleles, aber doch in einem gewissen Sinne vergleichbares Phänomen wird regelmäßig aus Befragungen zur wirtschaftlichen Lage berichtet. Dort zeigt sich, dass viele Befragte die allgemeine Lage eher negativ beurteilen, während die individuelle persönliche Situation deutlich positiver gesehen wird. Das kann etwa anhand der Daten des SOEP gezeigt werden: aktuell z. B. in Zeiten der Corona-Krise (vgl. Schröder et al. 2020: 12-14) oder während der Finanzmarktkrise 2008/2009 (vgl. Grabka, Goebel \& Liebig 2019).

17 Bei den Merkmalen Alter und formaler Bildungsabschluss erfolgte die Gruppenbildung mittels Medianhalbierung, bei dem binären Merkmal Geschlecht entlang der Werte „männlich“ (linker Balken) und „weiblich“ (rechter Balken). 


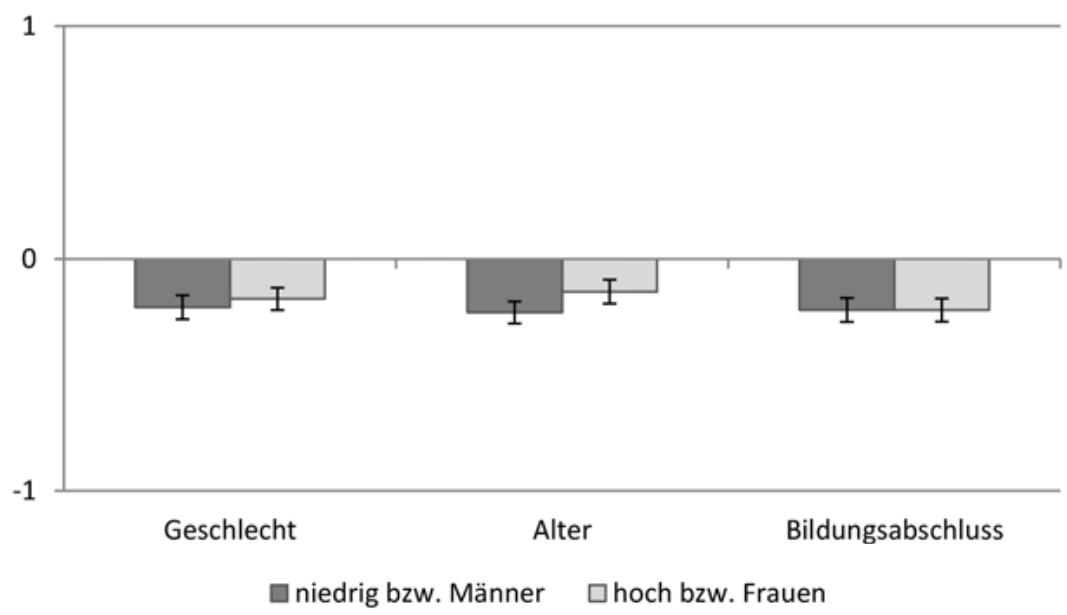

Abb. 6a: Bewertung der Entwicklung der deutschen Sprache nach soziodemographischen Faktoren (DE2017; $n_{\text {Männer }}=335, n_{\text {Frauen }}=376 ; n_{\text {Alter niedrig }}=381, n_{\text {Alter hoch }}=330, n_{\text {Bildungsabschluss nied- }}$ rig $=340, n_{\text {Bildungsabschluss hoch }}=321$; Mittelwerte mit abgeschnittener Achse und $-1=$,eher negativ“, $0=$,teils/teils“, 1=,eher positiv“; Frage wie Abb. 5).

Diese Darstellungsweise macht den Befund aus Abbildung 5 auf andere Weise sichtbar: insgesamt wird die Entwicklung der deutschen Sprache negativ beurteilt. Wie man sieht, liegen alle Balken deutlich unterhalb der Nulllinie, der Gesamtblick ist klar negativ. Alle Balken liegen in etwa auf einem Niveau; das bedeutet, dass es bei der Frage nach der Entwicklung der deutschen Sprache im Antwortverhalten der Befragten hinsichtlich ihrer soziodemographischen Merkmale keine wesentlichen Unterschiede gibt. Allenfalls beim Alter scheint es eine leichte Tendenz zu geben, dass die jüngeren Befragten eher noch skeptischer sind als die älteren, aber statistisch ist dies nicht signifikant.

Etwas anders verhält es sich bei der Teilstichprobe, die auf die Frage nach dem Zustand der deutschen Sprache geantwortet hat (Abb. 6b). 


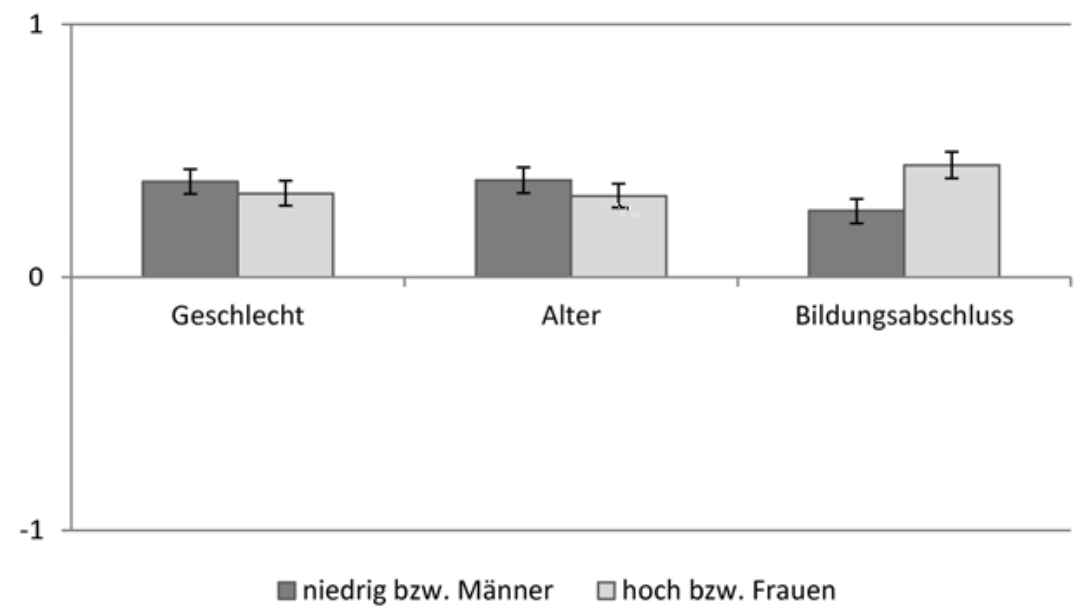

Abb. 6b: Bewertung des Zustands der deutschen Sprache nach soziodemographischen Faktoren (DE2017; $n_{\text {Männer }}=338, n_{\text {Frauen }}=372 ; n_{\text {Alter niedrig }}=365, n_{\text {Alter hoch }}=345, n_{\text {Bildungsabschluss niedrig }}=360$, $n_{\text {Bildungsabschluss hoch }}=309$; Mittelwerte mit abgeschnittener Achse und -1=,eher negativ“, $0=$,teils/teils“, 1=,eher positiv“; Frage wie Abb. 5).

Hier liegen alle Balken im positiven Bereich. Bei den Merkmalen Geschlecht und Alter sind die Unterschiede der Teilgruppen wiederum marginal. Ein klarer und statistisch signifikanter, wenn auch der Effektgröße nach eher kleiner Unterschied zeigt sich jedoch beim Bildungsabschluss: Befragte mit einem höheren formalen Bildungsabschluss geben im Schnitt insgesamt positive Bewertungen $a b$ als diejenigen mit einem niedrigeren Bildungsabschluss.

\section{Veränderungen}

Sprache ist einerseits stetem Wandel unterworfen, andererseits weist sie stets Variation auf (in Laiendiskursen wird bisweilen Letzteres mit Ersterem verwechselt). Die Tatsache, dass die Frage nach der Entwicklung der deutschen Sprache bei den Befragten keinerlei Irritationen auslöst (es gibt zu dieser Frage so gut wie keine fehlenden Antworten), deutet darauf hin, dass es durchaus ein weit verbreitetes Bewusstsein einer Dynamik im Sprachgebrauch gibt. Wie schon in der Deutschland-Erhebung 2008 haben wir die Wahrnehmung sprachlicher Veränderungen auch in der Deutschland-Erhebung 2017 explizit erfragt (Abb. 7). 


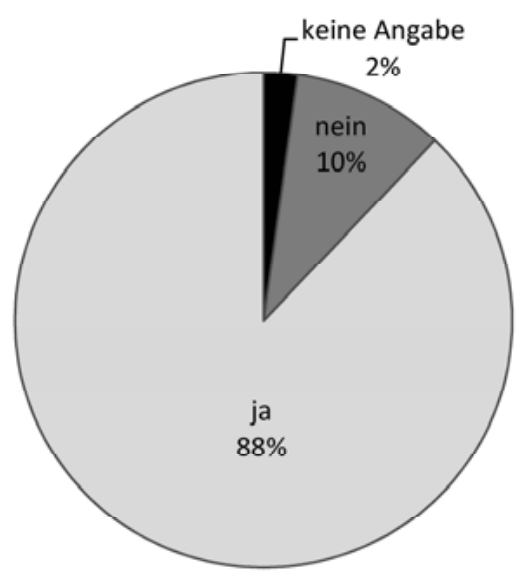

Abb. 7: Veränderungen in der deutschen Sprache (DE2017; N=1439; Frage: „Sind Ihnen in den letzten Jahren Veränderungen in der deutschen Sprache aufgefallen?“).

Die große Mehrheit der Befragten (88 \%) gibt an, tatsächlich solche Veränderungen wahrgenommen zu haben; nur jeder Zehnte will in den letzten Jahren keine sprachlichen Veränderungen bemerkt haben. ${ }^{18}$ Diejenigen, die angegeben haben, dass ihnen Veränderungen aufgefallen seien, wurden im Anschluss gefragt, wie stark sich nach ihrer Wahrnehmung die deutsche Sprache verändert habe (Abb. 8), und wurden dann gefragt, um welche Veränderungen es sich handele, und gebeten, Beispiele zu nennen (Abb. 9). Insgesamt wird der Sprache (oder dem Sprachgebrauch) durchaus ein hohes Maß an Dynamik zugestanden.

18 Dieses Ergebnis ähnelt sehr stark den Ergebnissen der Deutschland-Erhebung 2008; damals gaben $84 \%$ der Befragten an, sprachliche Veränderungen bemerkt zu haben; $15 \%$ beantworteten die entsprechende Frage mit „nein“ (vgl. Gärtig, Plewnia \& Rothe 2010: 195-197). 


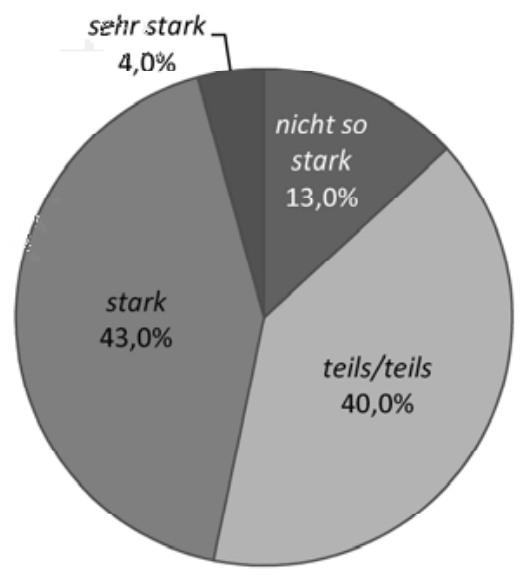

Abb. 8: Grad der Veränderungen in der deutschen Sprache (DE2017; N=1266; Frage: „Und wie stark hat sich die deutsche Sprache verändert?“, im Diagramm nicht sichtbar: „überhaupt nicht“: 0,2 \% und „keine Angabe“: 0,2\%).

Fast die Hälfte (47 \%) urteilt, die deutsche Sprache habe sich „stark“ oder „sehr stark“ verändert; $40 \%$ antworten mit „teils/teils“. $\mathrm{Zu}$ beachten ist, dass die Bezugsgröße hier nicht die gesamte Stichprobe ist, sondern nur diejenigen $88 \%$ der Befragten, die überhaupt angeben, dass ihnen Veränderungen aufgefallen sind (vgl. Abb. 7). Offenbar ist aber, wenn man sich entschlossen hat, die Frage nach der Existenz sprachlicher Veränderungen zu bejahen, ist die Bereitschaft groß, diese Veränderungen auch für relevant zu erklären.

Die beiden in Abbildung 7 und 8 besprochenen Fragen hatten in erster Linie Hinführungscharakter. Interessant für unseren Zusammenhang ist die anschließende Frage, welche konkreten sprachlichen Veränderungen von den Befragten benannt werden (Abb. 9). Die Frage war offen gestellt, d. h. die Befragten hatten keinerlei Vorgaben, sondern konnten - und mussten - ihre Antworten frei formulieren; es konnten mehrere Angaben gemacht werden. Solche offenen Fragen sind für die Probanden kognitiv wesentlich anspruchsvoller als geschlossene Listenformate, und sie stellen auch für die Auswertung eine gröBere Herausforderung dar, weil die Einzelantworten, die dann in der Ergebnisdarstellung zu Kategorien zusammengefasst werden müssen, unter Umständen sehr heterogen ausfallen und sich auf sehr unterschiedliche Ebenen beziehen können. Der große Vorteil dieser Vorgehensweise, die auf aktives Sprachwissen 
zielt, besteht aber darin, dass so ein Priming weitestgehend vermieden wird und man keine Echo-Antworten erhält. ${ }^{19}$

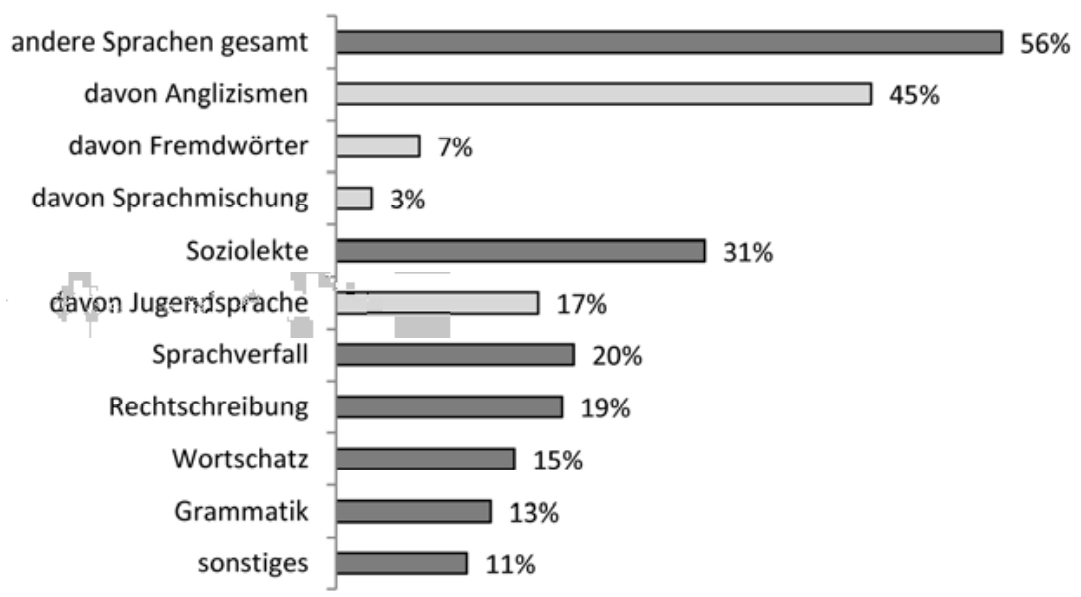

Abb. 9: Veränderungen in der deutschen Sprache nach Themenbereichen (DE2017; N=1439; Frage: „Was hat sich an der deutschen Sprache verändert? Fallen Ihnen da Beispiele ein?“).

Abbildung 9 zeigt die verschiedenen Antworten in sinnvollen Kategorien gruppiert; die Kategorien wurden induktiv nach Sichtung der Einzelwerte gebildet. Mehr als die Hälfte der Befragten (56 \%) nennt Veränderungen, die in der einen oder anderen Weise mit fremdsprachlichen Einflüssen auf das Deutsche zu tun haben; unter diesen nehmen Anglizismen (mit $45 \%$ ) eine herausgehobene Stellung ein; Anglizismen spielen offenbar im Bewusstsein vieler Sprecherinnen und Sprecher eine prominente Rolle. Eine allgemeine Zunahme von Fremdwörtern konstatieren nur $7 \%$ der Befragten. Migrantensprachen, wie z. B. Türkisch oder Russisch, werden als Gebersprache kaum genannt. Ein anderes Themencluster, dem sich die Antworten von einem knappen Drittel der Probanden zuordnen lassen, bezieht sich auf Soziolekte in einem weiten Sinne; Kommentare, die sich auf Jugendsprachliches beziehen, machen hier mit $17 \%$ die größte Untergruppe aus. Jeder fünfte Befragte bedient den eingangs besprochenen Sprachverfalls-Topos; das mag man angesichts der medialen Präsenz dieses Themas für wenig halten. Die Rechtschreibung wird ebenfalls nur von knapp

$19 \mathrm{Zu}$ den Vor- und Nachteilen der unterschiedlichen Frageformate vgl. z. B. Plewnia \& Rothe (2012: 27-33). 
jedem Fünften thematisiert. Weitere Nennungen behandeln, meistens punktuell und unscharf, lexikalische oder grammatische Themen. Auffällig ist, dass bestimmte Themen, die man vielleicht erwarten könnte, allenfalls marginal erscheinen: so spielt beispielsweise weder das Thema Leichte Sprache noch die Frage des sprachlichen Genderns, zu der es aktuell einen recht lebhaften öffentlichen Diskurs gibt, eine Rolle.

Wie verteilt sich nun die Nennung dieser verschiedenen Themenbereiche über die Stichprobe? In den folgenden Diagrammen präsentieren wir eine Auswahl der genannten Themen aufgeschlüsselt entlang der soziodemographischen Merkmale ,Geschlecht‘ (Abb. 10), ,Alter (Abb. 11) und ,formaler Bildungsabschluss‘ (Abb. 12).

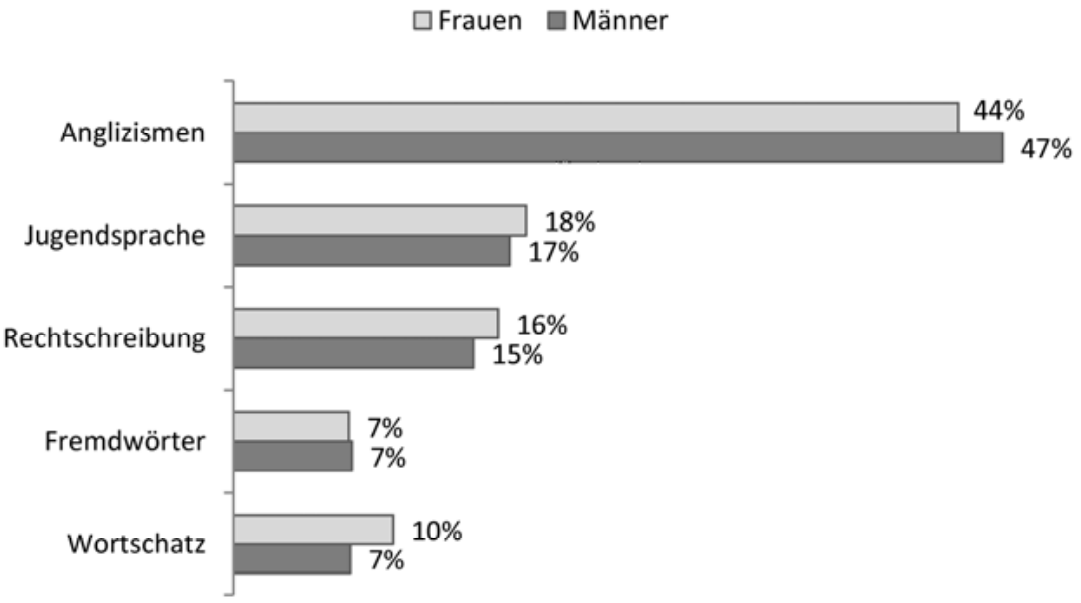

Abb. 10: Veränderungen (ausgewählte Themenbereiche) nach Geschlecht (DE2017; $n=760$ (Frauen), $n=679$ (Männer); Frage wie Abb. 9).

Bei der Unterscheidung nach Geschlecht (Abb. 10) sehen wir so gut wie keine Unterschiede. Einzig bei den auf den Wortschatz bezogenen Nennungen scheint es eine gewisse Geschlechterdifferenz zu geben, aber auch dieser Unterschied erweist sich bei der statistischen Prüfung als nicht signifikant.

Anders ist es hingegen beim Alter (Abb. 11), dort zeigen sich durchaus bestimmte Muster. 


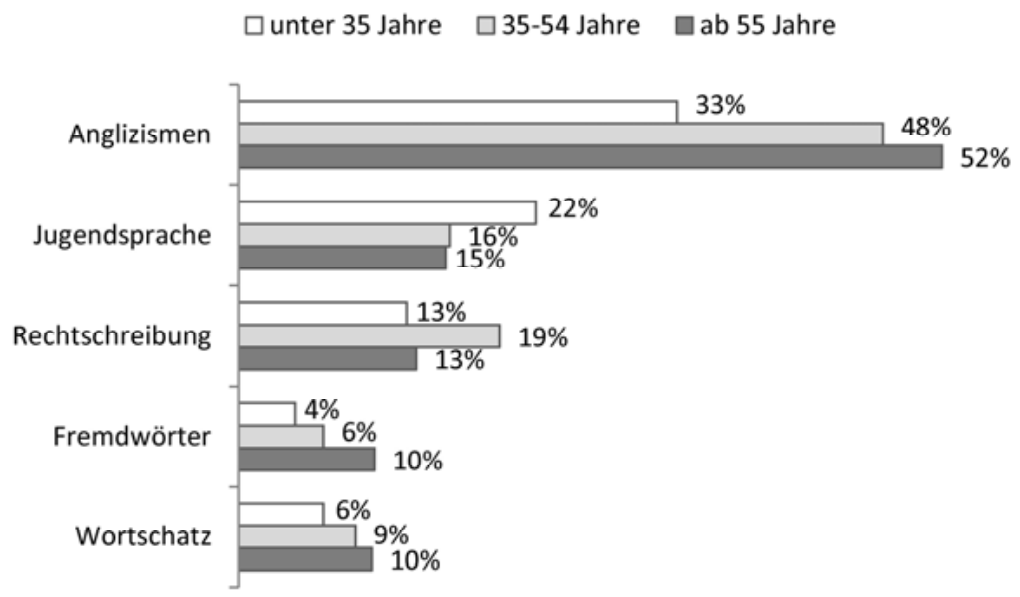

Abb. 11: Veränderungen (ausgewählte Themenbereiche) nach Alter (DE2017, $n=384$ (unter 35 Jahre), $n=541$ (35-54 Jahre), $n=514$ (ab 55 Jahre); Frage wie Abb. 9). ${ }^{20}$

Hier zeigt sich, dass das Anglizismen-Thema eines ist, das vor allem die ältere Generation bewegt; von den jüngeren Befragten werden Anglizismen signifikant seltener genannt. Umgekehrt - und das mag vielleicht überraschen - werden Veränderungen, die mit jugendsprachlichen Redeweisen in Zusammenhang gebracht werden, signifikant häufiger von den Befragten der jüngeren Generation thematisiert. Einen sehr deutlichen, statistisch signifikanten Alterseffekt gibt es auch beim Themenkomplex Fremdwörter, diese werden seltener von den jüngeren und häufiger von den älteren Befragten genannt. Der höchste Wert von Nennungen, die sich auf das Thema Rechtschreibung beziehen, findet sich bei den Befragten der mittleren Generation; das bedeutet, es ist die Generation der bei der Einführung der Rechtschreibreform 1996/1998 unter 35Jährigen. Möglicherweise zeigt sich hier ein Reflex der tatsächlichen Betroffenheit.

Auch beim Merkmal ,formaler Bildungsabschluss‘ (Abb. 12) lassen sich bestimmte Muster erkennen.

20 Die Befragten wurden nach ihren Geburtsjahren diesen drei etwa gleich große Altersspannen umfassenden Altersgruppen zugeordnet. 


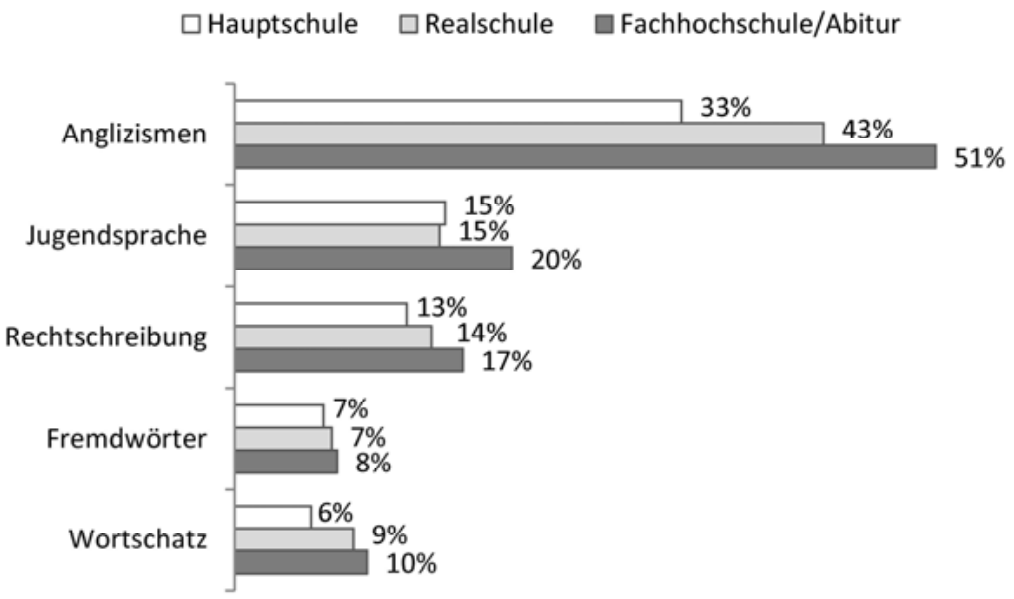

Abb. 12: Veränderungen (ausgewählte Themenbereiche) nach Bildungsabschluss (DE2017; $n=214$ (Hauptschule), $n=492$ (Realschule), $n=639$ (Fachhochschule und Abitur); Frage wie Abb. 9).

Sehr deutlich ist hier beim Anglizismen-Thema eine Treppe zu erkennen, nach der Anglizismen umso eher thematisiert werden, je höher der formale Bildungsabschluss der Befragten ist. Während von den Befragten mit Hauptschulabschluss nur jeder Dritte Anglizismen nennt, ist es bei den Befragten mit Abitur beziehungsweise Fachhochschulreife jeder Zweite. Im Prinzip gilt das mehr oder weniger stark ausgeprägt für fast alle Bereiche: Je höher der Bildungsabschluss ist, desto eher werden überhaupt Veränderungen angeführt.

\section{Anglizismen}

Offenkundig ist das Thema Anglizismen unter den Bereichen, die als für die Sprachentwicklung besonders relevant erachtet werden, derjenige, der für die Befragten die prominenteste Rolle spielt. Es erscheint daher lohnend, das Profil derjenigen Probanden, die von sich aus Anglizismen als Thema nennen, genauer in den Blick zu nehmen. Dazu teilen wir die Stichprobe in zwei Gruppen: diejenigen, die Anglizismen nennen, und diejenigen, die es nicht tun. Für die soziodemographischen Merkmale ,Alter ${ }^{\star}$ und ,formaler Bildungsabschluss ergibt sich folgendes Bild (Abb. 13): 


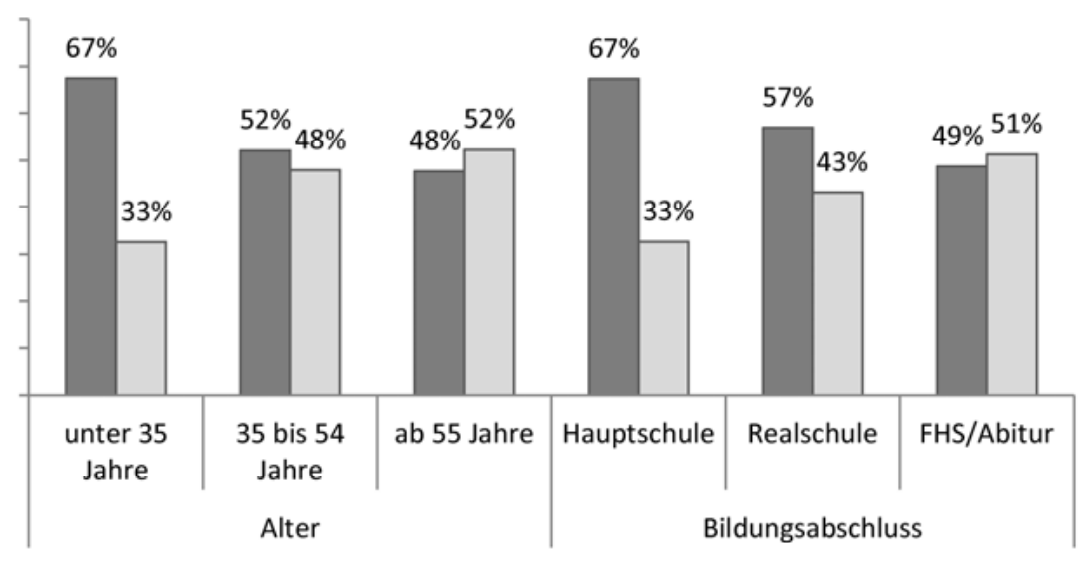

$\square$ keine Anglizismen $\square$ Anglizismen

Abb. 13: Nennung und Nicht-Nennung von Anglizismen nach Alter und Bildungsabschluss (DE2017; Teilstichproben wie Abb. 11 und 12; Frage wie Abb. 9).

Zum einen zeigt sich in Bezug auf das Alter noch augenfälliger, als es in Abbildung 11 der Fall war, dass das Thema Anglizismen die jüngeren Befragten deutlich weniger bewegt als die älteren: Von den unter 35-Jährigen nennen zwei Drittel keine Anglizismen, nur ein Drittel nennt sie. Bei den beiden anderen Altersgruppen ist das Verhältnis zwischen Nennung und Nicht-Nennung hingegen ziemlich ausgewogen.

Auch der in Abbildung 12 bereits ablesbare Effekt wird hier gut sichtbar: Personen, die Anglizismen nennen (jeweils der linke, dunkle Balken), haben häufiger einen höheren Schulabschluss als Befragte, die das nicht tun (jeweils der rechte, helle Balken). Das dürfte natürlich auch damit zu tun haben, dass Befragte mit höherer Schulbildung auf diese Frage generell mehr und ausführlicher antworten. ${ }^{21}$

Wir haben oben (Abb. 5 sowie Abb. 6a und Abb. 6b) gesehen, dass es einen Unterschied macht, ob man die Probanden nach ihrer Einschätzung der Entwicklung der deutschen Sprache fragt oder nach ihrem Zustand; die Entwicklung wird deutlich negativer gesehen als der Zustand. Anglizismen werden das hat auch der impressionistische Zugang über die Korpusanalyse (vgl.

21 Vermutlich ist dieser Faktor sogar der ausschlaggebende. Dazu passt auch, dass Personen, die Anglizismen nennen, zugleich mehr Sprachen als sympathisch nennen (auch Englisch) und sich mehr Schulfremdsprachen wünschen. 
Abb. 1) gezeigt - typischerweise in einem sprachkritischen Kontext diskutiert. Insofern wäre erwartbar, dass diejenigen, die bei den wahrgenommenen Sprachveränderungen Anglizismen zum Thema machen, eher sprachskeptischen Positionen zuneigen. Wir schlüsseln daher die Antworten auf die Frage nach Entwicklung bzw. Zustand der deutschen Sprache nach dem Kriterium, ob Anglizismen genannt werden oder nicht, auf (Abb. 14a und Abb. 14b).

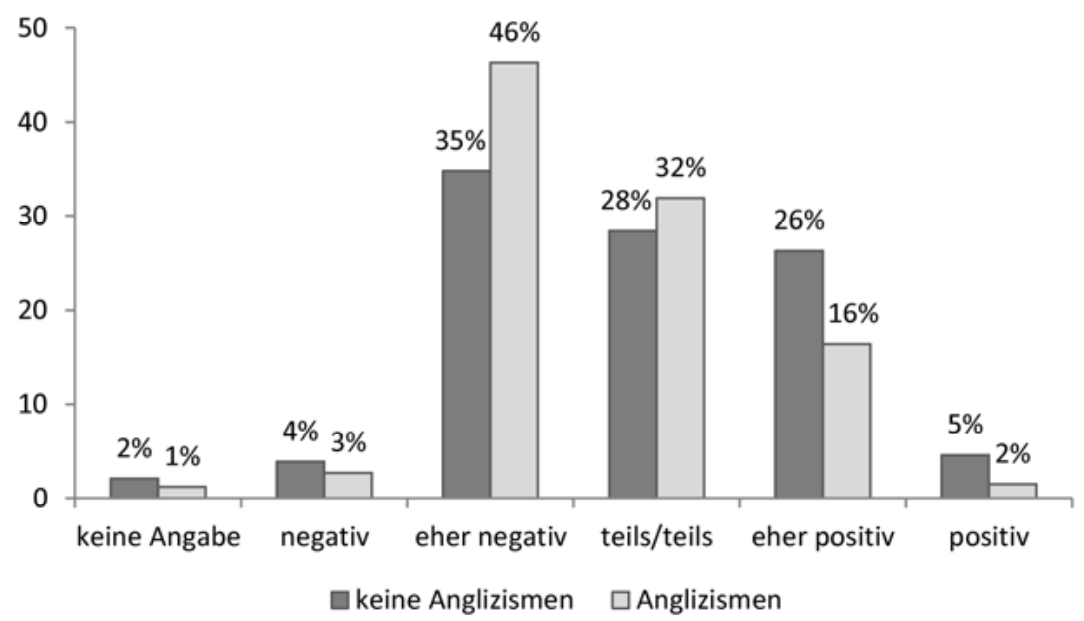

Abb. 14a: Nennung und Nicht-Nennung von Anglizismen nach der Bewertung der Entwicklung der deutschen Sprache (DE2017; Entwicklung: $n=388$ (keine Anglizismen), $n=335$ (Anglizismen)).

Für die Antworten auf die Frage nach der Entwicklung der deutschen Sprache zeigt sich der erwartete Zusammenhang tatsächlich: Diejenigen, die Anglizismen nennen, geben insgesamt deutlich negativere Einschätzungen ab als diejenigen, die Anglizismen nicht nennen. Anders sieht es hingegen bei der Teilstichprobe aus, die auf die Frage nach dem Zustand der deutschen Sprache geantwortet hat (Abb. 14b). 


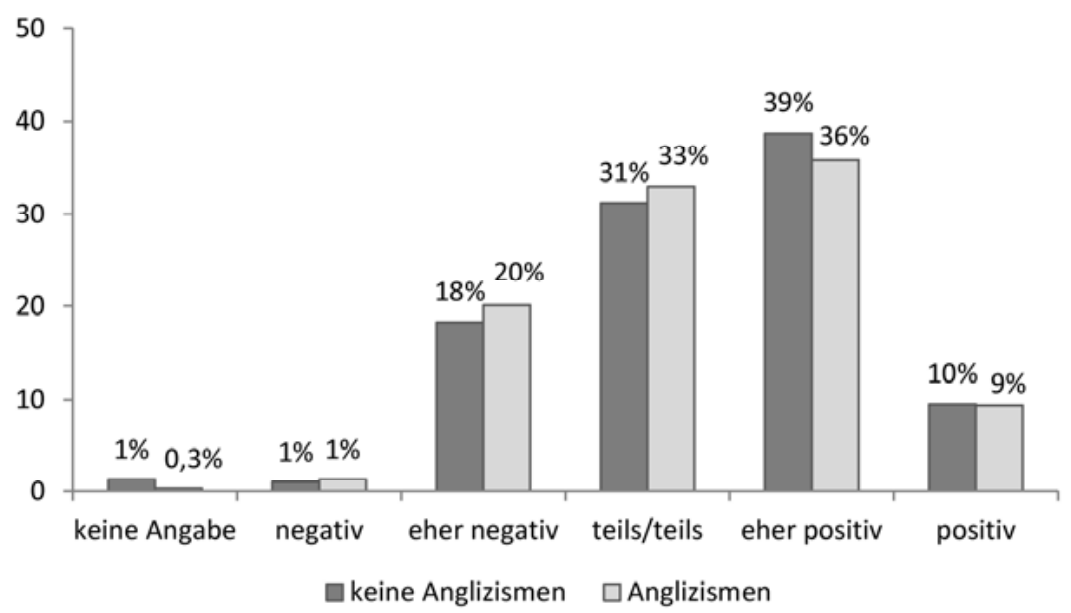

Abb. 14b: Nennung und Nicht-Nennung von Anglizismen nach der Bewertung des Zustands der deutschen Sprache (DE2017; $n=398$ (keine Anglizismen), $n=318$ (Anglizismen)).

Hier zeigt sich tatsächlich kein Effekt; es gibt keinen Zusammenhang zwischen der Nennung oder Nichtnennung von Anglizismen als Beispiel für Sprachveränderungen und der positiven oder negativen Bewertung des Zustands der deutschen Sprache.

\section{Andere Sprachen}

Während sich viele der Themen, die bei der Frage nach den wahrgenommenen sprachlichen Veränderungen genannt werden (vgl. Abb. 9), gewissermaßen nach innen richten - das gilt für den Diskurs über verschiedene Soziolekte ebenso wie für den Sprachrichtigkeitsdiskurs, in dem es um Fragen von Grammatik, Rechtschreibung und ähnlichem geht -, hat der Diskurs um Anglizismen viel mit Fremdheit zu tun. In diesem letzten Abschnitt wollen wir daher über einen bestimmten Ausschnitt der Wahrnehmung von Mehrsprachigkeit in der deutschen Gesellschaft berichten und diesen mit dem Anglizismen-Thema in Zusammenhang bringen.

Deutschland ist ein deutschsprachiges Land, das ist (auch ohne eine entsprechende verfassungsrechtliche Festlegung) unbestritten; ${ }^{22}$ in der Tradition

22 Zur rechtlichen Verankerung der deutschen Sprache in Deutschland vgl. Stickel (2012). 
des europäischen Nationalstaats-Konzepts sind Sprache und Staatlichkeit eng miteinander verbunden. Tatsächlich ist Deutschland aber keineswegs einsprachig, ${ }^{23}$ es gibt (wie in allen modernen westlichen Gesellschaften) einen durch verschiedene Formen von Migration steigenden Anteil von Personen mit einer anderen Muttersprache als Deutsch (neben den zahlenmäßig weniger ins Gewicht fallenden autochthonen Sprachminderheiten), was sich unter anderem in einem (in jüngerer Zeit dynamisierten) Diskurs um Migration und Mehrsprachigkeit sowie kulturelle und sprachliche Integration manifestiert. Dieser Mehrsprachigkeits-Anteil verändert die Gesellschaft; man könnte annehmen, dass sich die Präsenz anderer Sprachen auch in Sprachdiskursen in Bezug aufs Deutsche niederschlägt; wie wir gesehen haben, ist dies in nennenswertem Umfang nur für die Anglizismen der Fall. Nun ist der englisch-amerikanische Einfluss aufs Deutsche natürlich nicht auf große Zahlen englischsprachiger Einwanderer zurückzuführen, sondern auf die politische, ökonomische und kulturelle Hegemonie des angelsächsischen Raums. Gleichwohl gibt es hier eine thematische Verbindung über die sprachliche Alteritätserfahrung, die sowohl der konkret erlebbaren Alltagsmehrsprachigkeit als auch einer (typischerweise kritischen) Auseinandersetzung mit Anglizismen zugrunde liegt. Um ermessen zu können, welche subjektive Präsenz Mehrsprachigkeit für unsere Gewährspersonen hat, haben wir sie nach ihrer Vermutung bezüglich der Höhe des Anteils der Personen mit einer anderen Muttersprache als Deutsch an der Gesamtbevölkerung gefragt (Abb. 15).

23 Allerdings weiß man über die realen sprachlichen Verhältnisse in Deutschland erstaunlich wenig; vgl. Adler (2019). 


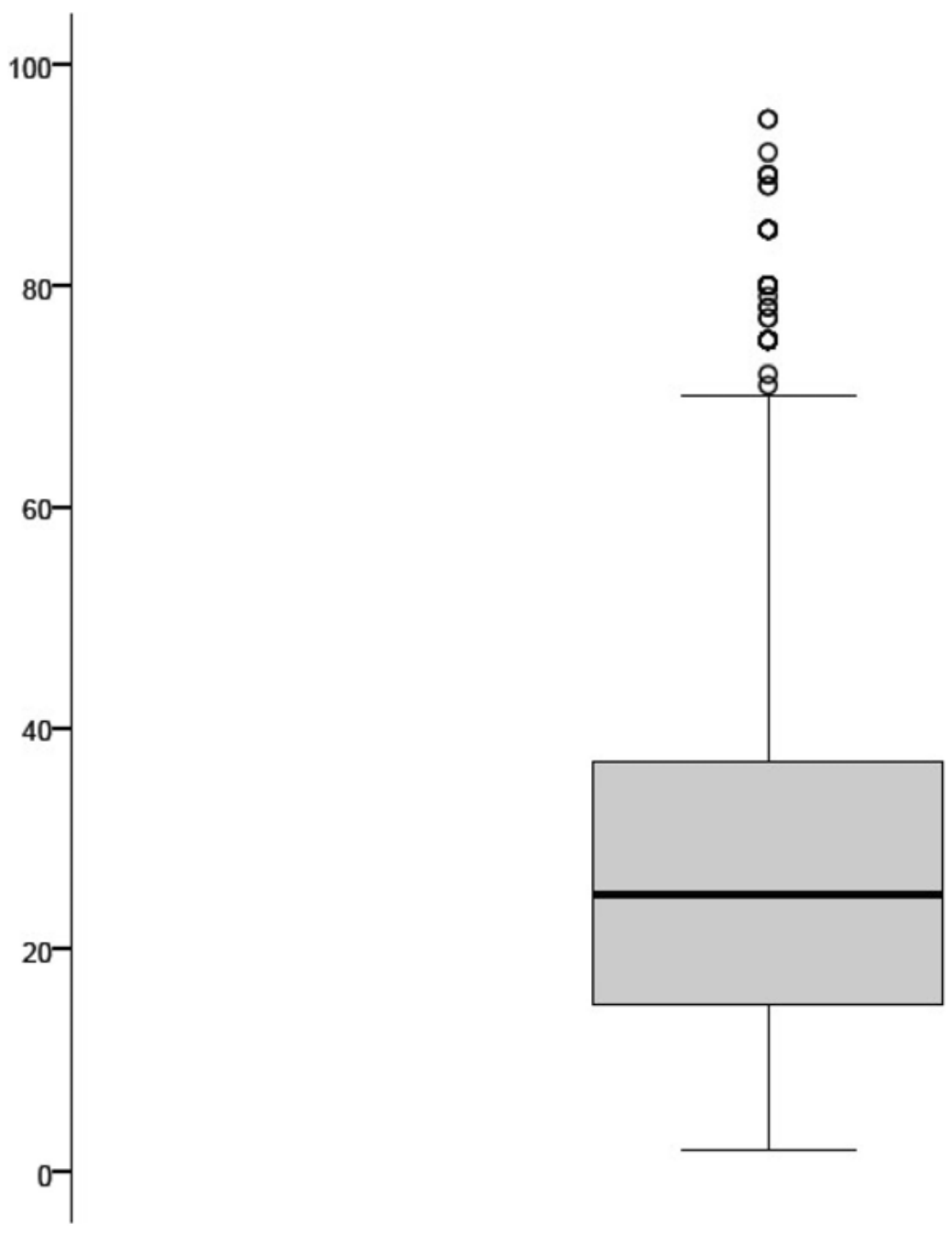

Abb. 15: Geschätzter Anteil anderer Muttersprachen als Deutsch (DE2017; N=1439; Median=24,5\%, Mittelwert=28,9\%; Frage: „Was schätzen Sie, wie viel Prozent der in Deutschland lebenden Bevölkerung hat eine andere Muttersprache als Deutsch?“).

Der Mittelwert der Schätzungen liegt bei 28,9 \%, der - wegen der verschiedenen Ausreißer hier vermutlich aussagekräftigere - Median bei 24,5\% (vgl. die dicke schwarze Linie im Boxplot). Gut jede vierte Person in Deutschland, so also die Schätzung unserer Probanden, hat eine andere Muttersprache als Deutsch. 
Auch hier lohnt sich der Blick auf verschiedene Teilgruppen entlang soziodemographischer Merkmale; hinzu kommt als Korrelat das Kriterium, ob auf die Frage nach Sprachveränderungen Anglizismen genannt werden oder nicht (Abb. 16).

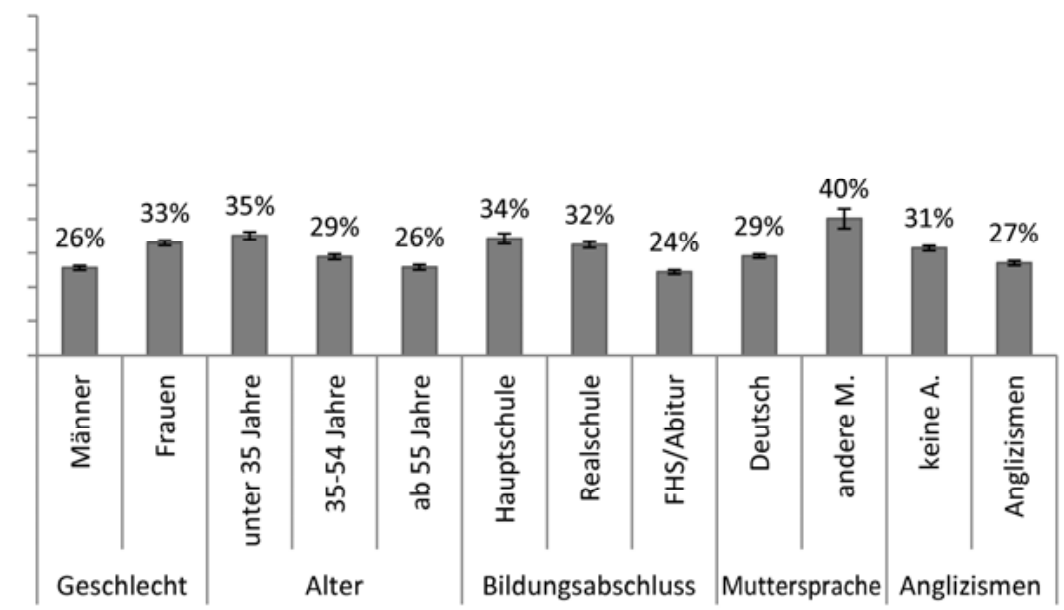

Abb. 16: Geschätzter Anteil von Personen mit einer andere Muttersprachen als Deutsch nach ausgewählten soziodemographischen Merkmalen (DE2017; $n_{\text {Männer }}=671, n_{\text {Frauen }}=739 ; n_{\text {unter }} 35$ Jahre $=382, n_{35-54 \text { Jahre }}=530, n_{\text {ab } 55 \text { Jahre }}=498 ; n_{\text {Hauptschule }}=210, n_{\text {Realschule }}=480, n_{\text {FHS } / \text { Abitur }}=626$, $n_{\text {Deutsch }}=1091, n_{\text {andere Muttersprache }}=57, n_{\text {keine Anglizismen }}=774, n_{\text {Anglizismen }}=636$; Frage wie Abb. 15 ).

Dabei unterscheiden sich die Einschätzungen der unterschiedlichen Gruppen bei allen betrachteten Merkmalen: Frauen schätzen den Anteil von Personen mit einer anderen Muttersprache als Deutsch signifikant höher ein als Männer. Auch ein Alterseffekt zeigt sich: je jünger die Befragten sind, desto höher veranschlagen sie den Anteil von Personen mit anderer Muttersprache. Das mag mit unterschiedlichen Wissensbeständen zusammenhängen, aber auch damit, dass sich die Lebenswelten der Generationen hier relevant unterscheiden. Eine ähnliche Treppe zeigt sich auch beim Merkmal ,formaler Bildungsabschluss': je höher die Schulbildung, desto niedriger die Schätzwerte. Ebenfalls signifikant sind die Unterschiede, wenn man die Stichprobe nach der Erstsprache der Befragten unterteilt: Personen, die selbst eine andere Muttersprache als Deutsch haben, schätzen den Anteil dieser Gruppe, zu der sie selbst gehören, deutlich höher ein als Befragte mit Deutsch als Muttersprache. Auch beim AnglizismenThema zeigt sich ein Unterschied: Personen, die bei den Sprachveränderungen 
Anglizismen nennen, setzen den Anteil von Personen mit nichtdeutscher Muttersprache niedriger an; dieser Befund korreliert auch mit den Daten zu Alter und Bildung.

Die Frage, wie richtig oder falsch die Befragten mit ihren Schätzungen liegen, ist gar nicht so einfach $\mathrm{zu}$ beantworten; wirklich belastbare Daten $\mathrm{zu}$ den in Deutschland gesprochenen Sprachen und ihrem jeweiligen Status gibt es nicht. Gewisse Annäherungen bieten einerseits unsere Deutschland-Erhebung 2017 und andererseits der Mikrozensus 2017 (Abb. 17).

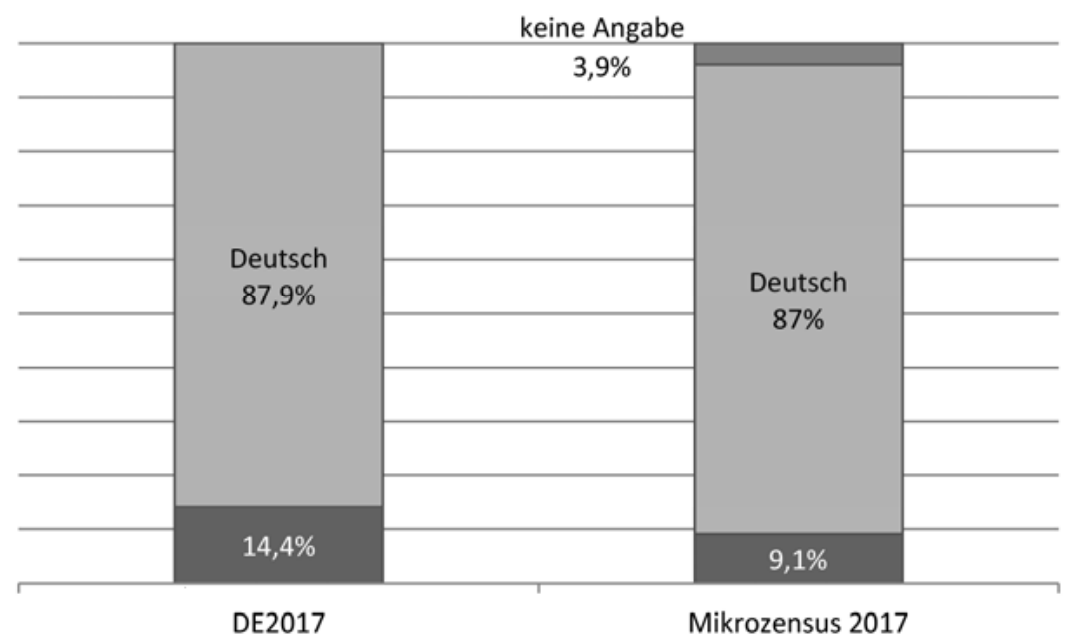

Abb. 17: Deutsch und andere Sprachen in Deutschland (DE2017; N=4.339; Frage: „Welche Sprache bzw. welche Sprachen würden Sie als Ihre Muttersprache bezeichnen?“, offene Frage, Mehrfachantworten möglich); Mikrozensus 2017 Haushaltssprachen; $N=41.304 .000$ Haushalte hochgerechnet auf der Basis von ca. 400.000 befragten Haushalten; Frage: „Welche Sprache wird in Ihrem Haushalt vorwiegend gesprochen?“, Haushaltsfrage, vorgegebenes Set an Antworten, Antwortpflicht, nur eine Person beantwortet die Frage). ${ }^{24}$

Laut Deutschland-Erhebung 2017 (der linke Balken in Abb. 17) haben $88 \%$ der Befragten Deutsch als Muttersprache, $14 \%$ geben eine andere oder eine weitere

24 Zur genauen Aufschlüsselung der offenen Frage der Deutschland-Erhebung 2017 vgl. Adler (2019: 213-214); für die Frage, die vorgegebenen Antworten und den Fragebogen im Mikrozensus 2017 vgl. Statistisches Bundesamt (2018a: 218); für die Ergebnisse des Mikrozensus 2017 vgl. Tabelle 18 in Statistisches Bundesamt (2018b: 484-492). 
Sprache als Muttersprache an. ${ }^{25}$ Dieser Wert liegt deutlich unter dem Durchschnitt der Schätzungen (Abb. 15). Vermutlich ist dieser Wert aber eher zu niedrig als zu hoch, denn obgleich die Stichprobe des SOEP-IS grundsätzlich repräsentativ ist, ist gerade im Bereich migrantischer Populationen Repräsentativität besonders schwer zu erreichen. Auf eine um ein Vielfaches größere Stichprobe kann der Mikrozensus zurückgreifen. Eine dort seit 2017 neu verankerte Frage adressiert nicht die Muttersprache, sondern die vorwiegend im Haushalt gesprochene Sprache (allerdings ist diese Frage in mehrerlei Hinsicht nicht ganz glücklich konstruiert, was die Aussagekraft der Ergebnisse stark einschränkt; vgl. dazu Adler 2019); hier (der rechte Balken in Abb. 17) geben $87 \%$ der Befragten Deutsch als dominierende Haushaltssprache an, nur $9 \%$ nennen eine andere Sprache. Auch dieser Wert dürfte - vor allem aufgrund der verschiedenen Unzulänglichkeiten im Fragedesign tatsächlich höher liegen. So oder so lässt sich aber festhalten, dass die Schätzungen der Gewährspersonen wohl alle deutlich zu hoch sind; der Anteil von Personen mit einer anderen Muttersprache als Deutsch an der Gesamtbevölkerung wird offenbar systematisch überschätzt. Über die tieferen Gründe dafür soll hier nicht spekuliert werden; der öffentliche Diskurs über Mehrsprachigkeit scheint aber zu gewissen Verzerrungen im Bewusstsein der Mehrheit der Befragten zu führen.

\section{Schluss}

Sprache ist eine unmittelbar alltagsrelevante Größe (vgl. ausführlich Hoffmeister in diesem Band) und als solche Gegenstand von Einstellungen und Bewertungen linguistischer Laien. Diese Einstellungen zu ermitteln, ist durchaus nicht trivial. Das Nachdenken über Sprache ist überwiegend geprägt von einer Einordnung in eine lange sprachkritische Tradition, die ihrerseits Teil eines Diskurses über einen allgemeinen Bildungs- und Sitten-Verfall ist; Sprachdiskurse werden daher im Allgemeinen als Negativdiskurse geführt. Die Gefahr, bei Fragebogenerhebungen, die die sprachlichen Befindlichkeiten linguistischer Laien zum Gegenstand haben, durch mehr oder weniger suggestive Frageformulierungen ungewollte Anknüpfungspunkte an diese Diskursmuster zu bieten, ist nicht zu unterschätzen. Etwas vereinfachend kann man sagen: Je neutraler die Frageformulierung, desto positiver die Ergebnisse; offenbar bildet der in öffentlich geführten Sprachdiskursen sehr präsente negative Zungenschlag nur einen

25 Die Summe ergibt mehr als $100 \%$, weil Mehrfachantworten möglich waren. 
Ausschnitt der Spracheinstellungswirklichkeit der Sprachgemeinschaft ab. Tatsächlich gibt es bestimmte sprachliche Themen, die die Sprecherinnen und Sprecher stärker bewegen, und andere, die in unseren aktuellen Daten nicht oder kaum genannt werden. Zu ersteren gehört zuvörderst - durchaus in Übereinstimmung mit dem öffentlich-medialen Diskurs - das Thema Anglizismen, das bei der Frage nach der Wahrnehmung sprachlicher Veränderungen das mit großem Abstand am häufigsten genannte Thema ist (womit über die tatsächlichen Verhältnisse des sprachlichen Wandels, das braucht hier nicht betont zu werden, selbstverständlich nichts ausgesagt ist). Zu letzteren gehört beispielsweise das Thema des sprachlichen Genderns, das zwar in jüngerer Zeit eine gewisse mediale Aufmerksamkeit genießt, das aber in unseren Daten praktisch keine Rolle spielt. Wir konstatieren hier eine Nichtübereinstimmung bestimmter Themen des öffentlich-medialen Diskurses und jener Themen, die für die Mehrheit der linguistischen Laien von Interesse sind.

Dass in unseren Daten bei der Frage nach den sprachlichen Veränderungen so oft das Thema Anglizismen genannt wird, legt nahe, dass die sprachliche Dynamik überwiegend als exogen interpretiert wird. Dabei zeigen sich hinsichtlich der verschiedenen Untergruppen der Stichprobe bestimmte soziodemographische Unterschiede (häufiger werden Anglizismen von Älteren genannt, häufiger von Personen mit höherer Schulbildung, keine Unterschiede gibt es beim Geschlecht). Dass die Wahrnehmung von Fremdheit stark von Subjektivität geprägt ist, belegt auch unsere Frage nach dem geschätzten Anteil von Personen mit einer anderen Muttersprache als Deutsch an der Gesamtbevölkerung. Dieser Anteil wird systematisch überschätzt, wobei es auch hier interessante soziodemographische Unterschiede gibt, die denjenigen, die im Zusammenhang mit der Nennung bzw. Nichtnennung von Anglizismen zu beobachten sind, ähneln. Verschiedene gesellschaftliche Gruppen schauen unterschiedlich auf die Welt und folglich auch unterschiedlich auf die Sprache; nicht immer decken sich die Wahrnehmungen und Bewertungen linguistischer Laien mit den Beschreibungen und Analysen der Sprachwissenschaft, und nicht immer passen sie zu den thematischen Gewichtungen des öffentlich-medialen Diskurses. Um hier einen Ausgleich zu schaffen, ist es unerlässlich, in einem ersten Schritt die Meinungen linguistischer Laien als legitime Positionen zur Kenntnis zu nehmen. 


\section{Literaturverzeichnis}

Adler, Astrid (2019): Sprachstatistik in Deutschland. Deutsche Sprache 19 (1), 197-219.

Adler, Astrid \& Karolina Hansen (2020): Sind Personen, die studieren, Studierende? Verwendungspräferenzen linguistischer Laien. In Andrea-Eva Ewels \& Albrecht Plewnia (Hrsg.), Themenheft Sprache und Geschlecht. Beiträge zur Gender-Debatte (Muttersprache 130 (1)), 47-63. Wiesbaden: Gesellschaft für deutsche Sprache.

Adler, Astrid \& Albrecht Plewnia (2018): Möglichkeiten und Grenzen der quantitativen Spracheinstellungsforschung. In Alexandra N. Lenz \& Albrecht Plewnia (Hrsg.), Variation - Normen - Identitäten, 63-98. Berlin, Boston: De Gruyter.

Eichinger, Ludwig M., Anne-Kathrin Gärtig, Albrecht Plewnia, Janin Roessel, Astrid Rothe, Selma Rudert, Christiane Schoel, Dagmar Stahlberg \& Gerhard Stickel (2009): Aktuelle Spracheinstellungen in Deutschland. Erste Ergebnisse einer bundesweiten Repräsentativumfrage. Mannheim: Institut für Deutsche Sprache/Universität Mannheim.

Eichinger, Ludwig M., Albrecht Plewnia, Christiane Schoel \& Dagmar Stahlberg (Hrsg.) (2012): Sprache und Einstellungen. Spracheinstellungen aus sprachwissenschaftlicher und sozialpsychologischer Perspektive. Mit einer Sprachstandserhebung zum Deutschen von Gerhard Stickel. Tübingen: Narr.

Eisenberg, Peter (2013): Anglizismen im Deutschen. In Deutsche Akademie für Sprache und Dichtung und Union der deutschen Akademien der Wissenschaften (Hrsg.), Reichtum und Armut der deutschen Sprache. Erster Bericht zur Lage der deutschen Sprache, 57-119. Berlin: De Gruyter.

Gärtig, Anne-Kathrin, Albrecht Plewnia \& Astrid Rothe (2010): Wie Menschen in Deutschland über Sprache denken. Ergebnisse einer bundesweiten Repräsentativerhebung zu aktuellen Spracheinstellungen. Mannheim: Institut für Deutsche Sprache.

Grabka, Markus M., Jan Goebel \& Stefan Liebig (2019): Wiederanstieg der Einkommensungleichheit: Aber auch deutlich steigende Realeinkommen, DIW-Wochenbericht 86 (19), 343-353. Berlin: Deutsches Institut für Wirtschaftsforschung (DIW).

Hoberg Rudolf, Karin M. Eichhoff-Cyrus \& Rüdiger Schulz (2008): Wie denken die Deutschen über ihre Muttersprache und über Fremdsprachen? Eine repräsentative Umfrage der Gesellschaft für deutsche Sprache. Wiesbaden: Gesellschaft für deutsche Sprache.

Lenzner, Timo \& Natalja Menold (2015): Frageformulierung. Mannheim: GESIS - LeibnizInstitut für Sozialwissenschaften. http://dx.doi.org/10.15465/gesis-sg_017.

Paulwitz, Thomas (2019): Zwischen Liebe und Sorge ums Deutsche. Deutsche Sprachwelt. Die Plattform für alle, die Sprache lieben 78, 3-9.

Pfalzgraf, Falco \& Torsten Leuschner (2006): Einstellungen zu Fremdwörtern und Anglizismen. Anmerkungen zu einer Repräsentativerhebung des Instituts für Deutsche Sprache. Germanistische Mitteilungen 64, 85-107.

Plewnia, Albrecht \& Astrid Rothe (2009): Eine Sprach-Mauer in den Köpfen? Über aktuelle Spracheinstellungen in Ost und West. In Albrecht Plewnia (Hrsg.), Sprache in Ost und West. Ein Themenheft zum 20. Jahrestag des Mauerfalls (Deutsche Sprache 37 (2/3), 235279. Berlin: Schmidt.

Plewnia, Albrecht \& Astrid Rothe (2012): Sprache - Einstellungen - Regionalität. In Ludwig M. Eichinger, Albrecht Plewnia, Christiane Schoel \& Dagmar Stahlberg (Hrsg.), Sprache und Einstellungen. Spracheinstellungen aus sprachwissenschaftlicher und sozialpsychologischer Perspektive, 4-134. Tübingen: Narr. 
Plewnia, Albrecht \& Andreas Witt (Hrsg.) (2014): Sprachverfall? Dynamik - Wandel - Variation. Berlin, Boston: De Gruyter.

Schröder, Carsten, Theresa Entringer, Jan Goebel, Markus M. Grabka, Daniel Graeber, Martin Kroh, Hannes Kröger, Simon Kühne, Stefan Liebig, Jürgen Schupp, Johannes Seebauer \& Sabine Zinn (2020): Erwerbstätige sind vor dem Covid-19-Virus nicht alle gleich. SOEPpapers on Multidisciplinary Panel Data Research 1080. Berlin: DIW Berlin. https://www.diw.de/documents/publikationen/73/diw_01.c.789529.de/diw_sp1080.pdf (letzter Zugriff 15.6.2020).

Spitzmüller, Jürgen (2020): Fremdwortdiskurs. In Thomas Niehr, Jörg Kilian \& Jürgen Schiewe (Hrsg.), Handbuch Sprachkritik, 218-224. Stuttgart: Metzler.

Statistisches Bundesamt (Destatis) (Hrsg.) (2018a): Bevölkerung und Erwerbstätigkeit. Haushalte und Familien. Ergebnisse des Mikrozensus 2017. Fachserie 1, Reihe 3. Statistisches Bundesamt.

Statistisches Bundesamt (Destatis) (Hg.) (2018b): Bevölkerung und Erwerbstätigkeit. Bevölkerung mit Migrationshintergrund. Ergebnisse des Mikrozensus 2017. Fachserie 1, Reihe 2. Statistisches Bundesamt.

Stickel, Gerhard \& Norbert Volz (1999): Meinungen und Einstellungen zur deutschen Sprache Ergebnisse einer bundesweiten Repräsentativerhebung. Mannheim: Institut für Deutsche Sprache.

Stickel, Gerhard (2012): Deutsch im Kontext anderer Sprachen in Deutschland heute: Daten und Einschätzungen. Unter Mitarbeit von Julia Weinheimer. In Ludwig M. Eichinger, Albrecht Plewnia, Christiane Schoel \& Dagmar Stahlberg (Hrsg.), Sprache und Einstellungen. Spracheinstellungen aus sprachwissenschaftlicher und sozialpsychologischer Perspektive, 227-321. Tübingen: Narr. 
